\title{
Optimal Therapeutic Control Modeling for Immune System Response
}

\author{
Pramila Bajpai \\ Department of Applied \\ Sciences, Vidya College of \\ Engineering, Meerut.
}

\author{
Ashish Chaturvedi \\ Director, Kishan Institute of \\ Engg \& Technology, Meerut.
}

\author{
A. P. Dwivedi \\ Director, MCA, Radha Govind \\ Engineering College, Meerut.
}

\begin{abstract}
Paper demonstrates the stochastic optimal control model to enhance immune system response. Immune system response can be amplified by agents that kill the pathogen, which stimulates the production of antibodies and implies the enhancement in the health of the organ. Imperfect measurements of the dynamic state degrade the precision of feedback adjustments to therapy; however, optimal state estimation allows the feedback strategy to be implemented with incomplete measurements and minimizes the expected effects of measurement error. The stochastic approach with genetic computing is evaluated to minimize the mutiobjective treatment cost function.
\end{abstract}

Key words: Immune system response, stochastic optimal control, Multi-objective cost function.

\section{INTRODUCTION}

Numerous reactive (protective) dynamic responsive mechanisms takes part to control the uncontrolled growth of pathogens when some infectious microbes strikes to the biological system. First, the resistant system of the body provides a unplanned response to the microbes, by killing pathogen if it can be, inducing inflammation and vasodilatation that aids the defense, causing blood coagulation that slows the spread of infection to other parts of the body, and raising the alarm for more complete response. In the process, a humoral response is initiated, signaling the presence of extracellular "non-self' organisms. This causes to activate Blood cells to convert in plasma cells that are specific to the intruders' antigens. The plasma cells produce antibodies that bind to the antigens, mediating the destruction of pathogens by various modalities [3-5]. The adaptive immune system provides a strategic response that is tailored to the primary attack, producing $\mathrm{B}$ and $\mathrm{T}$ cells, as well as a host of molecules, that defeat specific intracellular pathogens by binding to infected cells and either killing them outright, inducing programmed cell death, or signaling other cells to finish the job. Inherent, humoral, and adaptive immune responses can be coupled. Many models of immune response to infection have been already in literature [6], with recent emphasis on the human-immunodeficiency virus [7-8]. Norbert Wiener and Richard Bellman appreciated and anticipated the application of mathematical analysis to treatment in a broad sense can be seen in [9-10], and Swan surveyed early optimal control applications to biomedical problems. This can be seen in [11]. Optimal control theory was depicted as an organizing principle for natural immune system behavior in [12], and it is applied to HIV treatment in [13]. Intuitive control approaches are presented in [14]. The dynamics of drug response (pharmacokinetics) are modeled in [15], and control theory is applied to drug delivery is discussed in [16].

First, we evaluated remedial treatments with differing hypotheses about the initial pathogen concentration. If the initial concentration is known precisely [1], the optimizing control history maximizes efficacy of the drug while minimizing its side effects and cost. For the second study [2], a feedback strategy based on a linear perturbation model of response dynamics is derived to account for variations induced by unknown initial infection. The therapy is modified as a function of the difference between the optimal and observed dynamic states over the entire treatment period, assuming that the difference is measured without error. This paper deals a linear-optimal state evaluator in the feedback therapy to minimize the effects of measurement error. However, we are using the model of immune response that was employed in $[1,2]$.

\section{EVOLUTIONARY COMPUTING: GENETIC ALGORITHMS \& MULTI- OBJECTIVE GENETIC ALGORITHMS}

The concept of GA was developed by Holland and his colleagues in the 1960s and 1970s [17]. GA are inspired by the evolutionist theory explaining the origin of species. In nature, weak and unfit species within their environment are faced with loss by natural selection. The strong ones have greater opportunity to pass their genes to future generations via reproduction. In the long run, species carrying the correct combination in their genes become dominant in their population. Sometimes, during the slow process of evolution, random changes may occur in genes. If these changes provide additional advantages in the challenge for survival, new species evolve from the old ones. Unsuccessful changes are eliminated by natural selection. In a population-based approach, GA are one of the best tool to solve multi-objective optimization problems [27].

A generic single-objective GA can be modified to find a set of multiple non-dominated solutions in a single run. The ability of GA to simultaneously search different regions of a solution space makes it possible to find a diverse set of solutions for difficult problems with non-convex, discontinuous, and multimodal solutions spaces. The crossover operator of GA may exploit structures of good solutions with respect to different objectives to create new non-dominated solutions in unexplored parts of the Pareto front. In addition, most multi-objective GA do not require the user to prioritize, scale, or weigh objectives. 
Therefore, GA have been the most popular heuristic approach to multi-objective design and optimization problems. Jones et al. [18] reported that $90 \%$ of the approaches to multi-objective optimization aimed to approximate the true Pareto front for the underlying problem. A majority of these used a metaheuristic technique, and $70 \%$ of all metaheuristics approaches were based on evolutionary approaches.

The first multi-objective GA, called vector evaluated GA (or VEGA), was proposed by Schaffer [19]. Afterwards, several multi-objective evolutionary algorithms were developed including Multi-objective Genetic Algorithm (MOGA) [20], Weight-based Genetic Algorithm (WBGA) [21], Random Weighted Genetic Algorithm (RWGA) [22], Rank-Density Based Genetic Algorithm (RDGA) [23], Dynamic Multiobjective Evolutionary Algorithm (DMOEA) [24] etc.

\section{MATHEMATICAL MODELING OF IMMUNE SYSTEM RESPONSE}

We are considering here, the mathematical model as employed in $[1,2]$ which is the idealize model of a generic humoral immune response. The model consisting of four components: the concentration of a foreign pathogen $\left(\mathrm{y}_{1}\right)$, concentration of plasma cells $\left(\mathrm{y}_{2}\right)$, concentration of antibodies that bind to the antigen $\left(\mathrm{y}_{3}\right)$ and a measure of the health of an organ $\left(\mathrm{y}_{4}\right)$ that may be damaged in infection attack. The model presented in [1, 2] has not been accounted for therapy. We have modified the original model by adding active and passive immunotherapeutic control agents, $\mu_{i}$ (Active immunotherapy strengthens natural immune response, as by enhancing plasma cell and antibody production, while passive immunotherapy addresses the effects of infection directly, as in killing the pathogen or healing the infected organ) and an exogenous input, $v_{i}$, to the model: pathogen killer $\left(\mu_{1}\right)$, plasma cell booster $\left(\mu_{2}\right)$, antibody booster $\left(\mu_{3}\right)$, and organ healing booster $\left(\mu_{4}\right)$. The dynamic system can be represented by the following set of ordinary differential equations:

$$
\begin{aligned}
& y_{1}^{\prime}=\left(q_{11}-p_{12} y_{3}\right) y_{1}+q_{1} \mu_{11} \\
& y_{2}^{\prime}=p_{21}\left(y_{4}\right) p_{22} y_{3} y_{1}-p_{23}\left(y_{2}-y_{2}^{\prime}\right)+q_{2} \mu_{2} \\
& y_{3}^{\prime}=p_{31} y_{2}-\left(p_{32}+p_{33} y_{1}\right) y_{3}+q_{3} \mu_{3} \\
& y_{4^{\prime}}=\left(p_{41} y_{1}-p_{42} y_{4}+q_{4} \mu_{4}\right.
\end{aligned}
$$

The parameters used for this study are:

$$
\begin{aligned}
& p_{11}=p_{12}=p_{23}=p_{31}=p_{42}=q_{2}=q_{3}=2 ; \\
& q_{1}=q_{4}=-1 ; \\
& p_{33}=p_{41}=1.5 \\
& p_{22}=5 ; p_{32}=2.5
\end{aligned}
$$

Figure 2 shows typical uncontrolled response to increasing levels of pathogen concentration at the start of the time domain. We may assume some initial period of microbial infection and growth prior to beginning the simulated immune response at zero time.

\section{STOCHASTIC OPTIMAL THERAPEUTIC CONTROL MODEL \& ITS SOLUTION WITH GA}

The optimal therapeutic protocol is derived by minimizing a treatment cost function, $T H$ that punishes large values of pathogen concentration, poor organ health, and excessive application of therapeutic agents. This multi-objective, positivedefinite scalar cost function of many variables allows tradeoffs between important factors to be adjusted through the relative weighting of individual components. Systematic responses tend to reinforce each other while conflicting responses compete in the development of an optimal regimen. The cost function is evaluated over the fixed time interval $\left[\mathrm{t}_{\mathrm{i}}, \mathrm{t}_{\mathrm{f}}\right]$ and can be given as,

$$
\begin{aligned}
& \operatorname{TH}_{i}(y)=\frac{1}{2}\left(a_{11} y_{1_{f}}^{2}+a_{44} y_{4_{f}}^{2}\right) \\
& +\frac{1}{2} \int_{t_{i}}^{t_{f}}\left(b_{11} y_{1}^{2}+b_{44} y_{4}^{2}+c_{11} u_{1}^{2}+c_{22} u_{2}^{2}+c_{33} u_{3}^{2}+c_{44} u_{4}^{2}\right) d t
\end{aligned}
$$

The cost-function elements are squared to amplify the effects of large variations and to de-emphasize contributions of small variations. Each squared element is multiplied by a coefficient $\left(a_{i i} \quad \boldsymbol{b}_{i \boldsymbol{i}}\right.$ or $\left.\boldsymbol{c}_{i i}\right)$ that establishes the relative importance of the factor in the treatment cost. These coefficients could reflect financial cost of treatment, or they could represent physiological 'cost' such as virulence, toxicity, or discomfort. The resulting treatment protocol balances speed, efficacy, and cost of treatment against implicit side effects.

Being a population-based approach, GA are well suited to solve multi-objective optimization problems. The classical approach to solve a multi-objective optimization problem is to assign a weight $w_{i}$ to each normalized objective function $T H_{i}(y)$ so that the problem is converted to a single objective problem with a scalar objective function as follows,

$$
\begin{aligned}
& \min T H(y)=w_{1} T H_{1}^{\prime}(y)+w_{2} T H_{2}^{\prime}(y) \\
& +w_{3} T H_{3}^{\prime}(y)+\ldots \ldots \ldots \ldots \ldots \ldots+w_{k} T H_{k}^{\prime}(y)
\end{aligned}
$$

where $T H_{i}^{\prime}(y)$ the is the normalized objective function $T H_{i}(y)$ and $\sum w_{i}=1$. This approach is called the priori approach since the user is expected to provide the weights. Solving a problem with the objective function (7) for a given weight vector $w=\left\{w_{1}, w_{2}, w_{3}, \ldots \ldots ., w_{k}\right\}$ yields a single solution, and if multiple solutions are desired, the problem must be solved multiple times with different weight combinations. The main difficulty with this approach is selecting a weight vector for each run. To automate this process; Hajela and Lin [21] proposed the WBGA for multi-objective optimization (WBGA-MO) in the WBGA-MO, each solution $y_{i}$ in the population uses a different weight vector $w=\left\{w_{1}, w_{2}, w_{3}, \ldots \ldots ., w_{k}\right\}$ in the calculation of the 
summed objective function (6). The weight vector $w_{i}$ is embedded within the chromosome of solution $y_{i}$. Therefore, multiple solutions can be simultaneously searched in a single run. In addition, weight vectors can be adjusted to promote diversity of the population.

Other researchers [22,25] have proposed a MOGA based on a weighted sum of multiple objective functions where normalized weight vector $w_{i}$ is randomly generated for each solution $y_{i}$ during the selection phase at each generation. This approach aims to stipulate multiple search directions in a single run without using additional parameters. The general procedure of the RWGA using random weights is given as follows [25]:

Procedure RWGA:

$\mathrm{E}=$ external archive to store non-dominated solutions found during the search so far;

$n_{E}=$ number of elitist solutions immigrating from $\mathrm{E}$ to $\mathrm{P}$ in each generation.

Step 1: Generate a random population.

Step 2: Assign a fitness value to each solution $y \in P_{t}$ by performing the following steps:

Step 2.1: Generate a random number $u_{k}$ in $[0,1]$ for each objective $k, k=1, \ldots . ., \mathrm{K}$.

Step 2.2: Calculate the random weight of each objective $k$ as $w_{k}=\frac{1}{u_{k}} \sum_{i=1}^{k} u_{i}$.

Step 2.3: Calculate the fitness of the solution as $f(y)=\sum_{k=1}^{K} w_{k} z_{k}(y)$

Step 3: Calculate the selection probability of each solution $y \in P_{t}$ as follows:

$p(y)=\left(f(y)-f^{\min }\right)^{-1} \sum_{y \in P_{t}}\left(f(y)-f^{\min }\right)$

where $f^{\min }=\min \left\{f(y): y \in P_{t}\right\}$

Step 4: Select parents using the selection probabilities calculated in Step 3. Apply crossover onthe selected parent pairs to create $N$ offspring.Mutate offspring with a predefined mutation rate. Copy all offspring to $P_{t+1}$.Update $\mathrm{E}$ if necessary.

Step 5: Randomly remove $\mathrm{nE}$ solutions from $P_{t+1}$ and add the same number of solutions from E to $P_{t+l}$.

Step 6: If the stopping condition is not satisfied, set $t=t+1$ and go to Step 2. Otherwise, return to E.

The main advantage of the weighted sum approach is a straightforward implementation. Since a single objective is used in fitness assignment, a single objective GA can be used with minimum modifications. In addition, this approach is computationally efficient. The main disadvantage of this approach is that not all Pareto-optimal solutions can be investigated when the true Pareto front is non-convex.
Therefore, multi-objective GA based on the weighed sum approach have difficulty in finding solutions uniformly distributed over a non-convex tradeoff surface [26].

\section{CONCLUSION}

The model presented here having active and passive immunotherapeutic control agents, and pathogen killer, plasma cell booster, antibody booster, and organ healing booster to model enhanced immune system response. For a strong enough attack, the combination of immune response and nominal therapy is insufficient, and the pathogen grows without bound, killing the organ. The therapeutic protocol must be adjusted to accommodate the change, either through continued reevaluation of the stochastic optimal policy or through a simpler mechanism for modifying the policy in proportion to deviations from the expected response history.

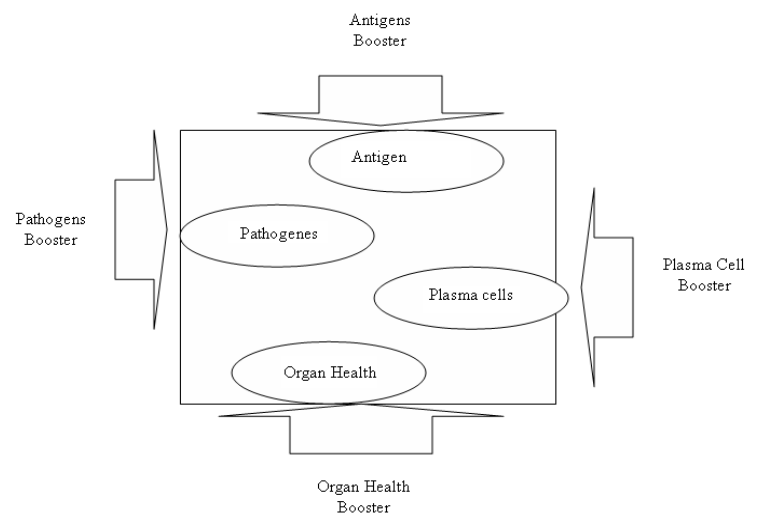

Figure 1: Proposed generic humoral immune system model
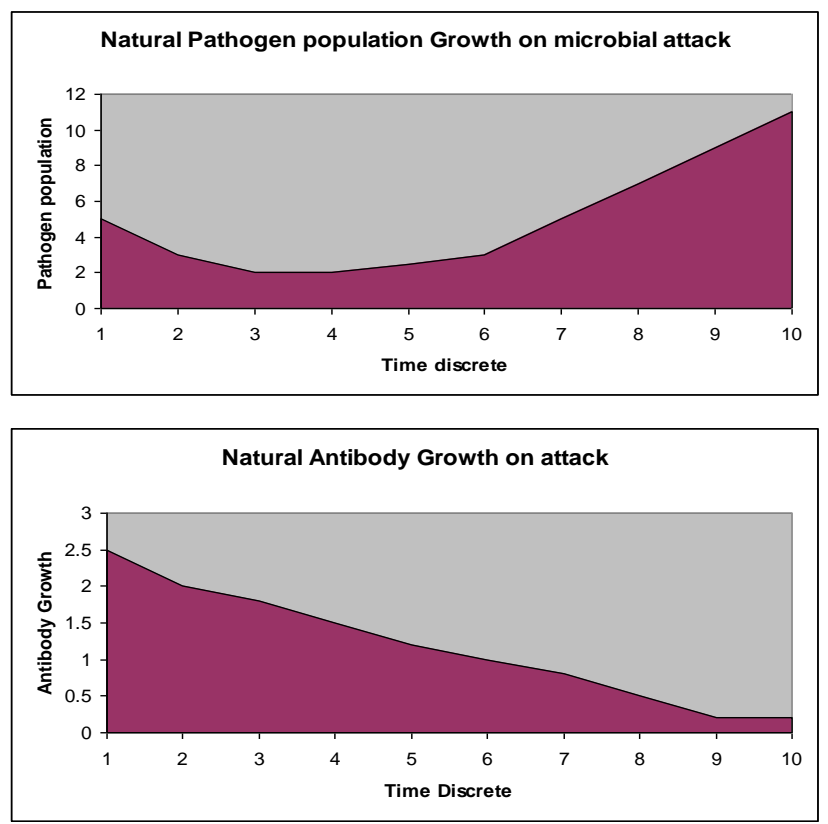

Figure 2: Pathogen and antibody response on microbial attack 

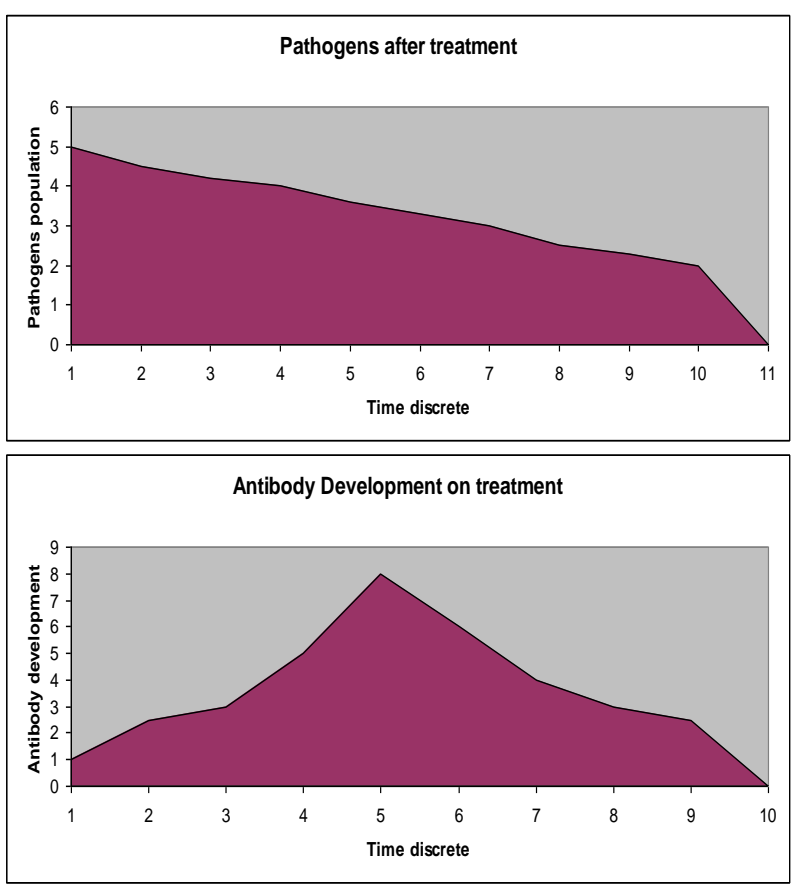

Figure 3 : shows the enhancement in immune system response after optimal therapy treatment.

\section{REFERENCES}

[1] R.F. Stengel, R. Ghigliazza, N. Kulkarni, O. Laplace, Optimal control of innate immune response, Optimal Contr. Appl. Methods 23 (2002) 91

[2] R.F. Stengel, R. Ghigliazza, N. Kulkarni, Optimal enhancement of immune response, Bioinformatics 18 (2002) 1227

[3] C.A. Janeway, P. Travers, M. Walport, M. Shlomchik, Immunobiology, Garland, London, 2001.

[4] P.M. Lydyard, A. Whelan, M.W. Fanger, Instant Notes in Immunology, Springer, New York, 2000.

[5] M. Thain, M. Hickman, The Penguin Dictionary of Biology, Penguin Books, London, 2000.

[6] M.A. Nowak, R.M. May, Virus Dynamics: Mathematical Principles of Immunology and Virology, Oxford University, Oxford, 2000.

[7] A.S. Perelson, P.W. Nelson, Mathematical analysis of HIV1 dynamics in vivo, SIAM Rev. 41 (1999) 3.

[8] M.A. Stafford, Y. Cao, D.D. Ho, L. Corey, A.S. Perelson, Modeling plasma virus concentration and CD4+ $\mathrm{T}$ cell kinetics during primary HIV infection, J. Theor. Biol. 203 (2000) 285.

[9] N. Wiener, Cybernetics: or Control and Communication in the Animal and the Machine, Technology, Cambridge, 1948.

[10] R.E. Bellman, Mathematical Methods in Medicine, World Scientific Press, Singapore, 1983.
[11] G.W. Swan, Role of optimal control theory in cancer therapy, Math. Biosci. 101 (1990) 237.

[12] A.S. Perelson, Applications of optimal control theory to immunology, in: R.R. Mohler, A. Ruberti (Eds.), Recent Developments in Variable Structure Systems Economics and Biology, Springer, Berlin, 1978, p. 272.

[13] L.M. Wein, S.A. Zenios, M.A. Nowak, Dynamic multidrug therapies for HIV: a control theoretic approach, J. Theor. Biol. 185 (1997) 15.

[14] D. Wodarz, K.M. Page, R.A. Arnaout, A.R. Thomsen, J.D. Lifson, M.A. Nowak, A new theory of cytotoxic Tlymphocyte memory: implications for HIV treatment, Philos. Trans. Roy. Soc. B 355 (2000) 329.

[15] J.M. van Rossum, O. Steyger, T. van Uem, G.J. Binkhorst, R.A.A. Maes, Pharmacokinetics by using mathematical systems dynamics, in: J. Eisenfeld, M. Witten (Eds.), Modelling of Biomedical Systems, Elsevier Science Publishers, 1986, p. 121.

[16] A. Gentilini, M. Morari, C. Bieniok, R. Wymann, T. Schnider, Closed-loop control of analgesia in humans, in: Proc. IEEE Conf. Decision and Control, Orlando, 2001, p. 861.

[17] J.H. Holland. Adaptation in natural and artificial systems. Ann Arbor: University of Michigan Press; 1975.

[18] D.F. Jones DF, S.K.Mirrazavi, M. Tamiz. Multiobjective meta-heuristics: an overview of the current state-of-the-art. Eur J Oper Res 2002;137(1):1-9.

[19] J.D. Schaffer. Multiple objective optimization with vector evaluated genetic algorithms. In: Proceedings of the international conference on genetic algorithm and their applications, 1985.

[20] C.M. Fonseca, P.J. Fleming. Multiobjective genetic algorithms. In: IEEE colloquium on 'Genetic Algorithms for Control Systems Engineering' (Digest No. 1993/130), 28 May 1993. London, UK: IEE; 1993.

[21] P. Hajela, C. lin. Genetic search strategies in multicriterion optimal design. Struct Optimization 1992;4(2):99-107.

[22] T. Murata, H. Ishibuchi. MOGA: multi-objective genetic algorithms. In: Proceedings of the 1995 IEEE international conference on evolutionary computation, 29 November-1 December, 1995. Perth, Australia: IEEE; 1995.

[23] H. Lu, G.G. Yen. Rank-density-based multiobjective genetic algorithm and benchmark test function study. IEEE Trans Evol Comput 2003;7(4):325-43.

[24] G.G. Yen, H. Lu. Dynamic multiobjective evolutionary algorithm: adaptive cell-based rank and density estimation. IEEE Trans Evol Comput 2003;7(3):253-74.

[25] T. Murata, H. Ishibuchi, H. Tanaka. Multi-objective genetic algorithm and its applications to flowshop scheduling. Comput Ind Eng 1996;30(4):957-68.

[26] E. Zitzler, K. Deb, L. Thiele. Comparison of multiobjective evolutionary algorithms: empirical results. Evol Comput 2000;8(2):173-95.

[27] C.A. Coello, 2005 http://www.lania.mx/ ccoello/ EMOO/EMOObib.html, 